\title{
$\nabla$ Association of Vitamin D Status with Cardiovascular Risk in Prediabetes
}

\section{IJCRR}

Section: Healthcare ISI Impact Factor (2019-20): 1.628

IC Value (2019): 90.81 $\operatorname{SJIF}(2020)=7.893$

(c) (7) (8)

Copyright@IJCRR

\section{Vidya Bhakta ${ }^{1 *}$, Said Khader ${ }^{2}$, Rubeena Quadri ${ }^{3}$}

\author{
'MBBS, MD, DNB; Department of Laboratory Medicine; Dr Sulaiman Al Habib Medical Group, Olaya Medical Complex, Riyadh, KSA; ${ }^{2} \mathrm{MBChB}$, \\ MRCP (UK), FRCPE; Department of Endocrinology, Dr Sulaiman Al Habib Medical Group, Olaya Medical Complex, Riyadh, KSA; ${ }^{3}$ MBBS, MD, \\ Department of Internal Medicine, Dr. Sulaiman Al Habib Medical Group, Olaya Medical Complex, Riyadh, KSA.
}

\section{ABSTRACT}

Introduction: Vitamin D (VD) has a key role in several metabolic processes and its deficiency may have adverse health implications. The study focuses on prediabetic patients and the impact of VD status on their cardiovascular health.

Aims: To determine the association between VD status and cardiovascular risk in prediabetic patients.

Methods: The study included retrospectively collected data on 270 prediabetic patients and 299 normoglycemic subjects. Prediabetes was diagnosed by $\mathrm{HbA} 1 \mathrm{c}$ as $5.7-6.4 \%$. Increased cardiovascular risk was assessed based on the Atherogenic Index of Plasma (AIP) more than 0.1. Lipid parameters and AIP were compared between two groups by t-test and chi-square and separately by ANOVA under VD categories; Sufficient $(>75 \mathrm{nmol} / \mathrm{L})$, Insufficient $(50-75 \mathrm{nmol} / \mathrm{L})$ and Deficient $(<50 \mathrm{nmol} / \mathrm{L})$. Regression analysis was used to find the association of VD status with risk of prediabetes and unfavourable AIP $(>0.1)$.

Results: VD deficiency was more prevalent in prediabetic subjects $(56.9 \% ; 57.9+20.8 \mathrm{nmol} / \mathrm{L} ; \mathrm{p}<0.05)$. AIP was significantly higher in prediabetic subjects $(0.13+0.29 ; p<0.05)$. AlP showed a significant increasing trend with declining Vitamin $D$ status among prediabetic patients $(p<0.05)$ but not in normoglycemic subjects. The risk of prediabetes $(p=0.141)$ and unfavourable AIP $(p<0.05)$ decreased with improved Vitamin $D$ status.

Conclusion: Vitamin D status is important in prediabetes as it potentially increases cardiovascular risk in the already vulnerable group.

Key Words: Prediabetes, Vitamin D, Atherogenic Index of Plasma, Cardiovascular disease, Dyslipidemia

\section{INTRODUCTION}

Evidence propounds that VD has an inevitable role in several metabolic processes and its deficiency is associated with hypertension, dyslipidemia, diabetes, hypertension and obesity. ${ }^{1}$ Prediabetes regardless of progressing to type 2 diabetes mellitus still poses a high risk of associated prolonged damages like increased cardiovascular risk and neural damage. ${ }^{2}$ Prediabetic patients usually have lower VD compared to those with normal glucose tolerance ${ }^{3}$ and also, the risk of progressing to diabetes increases with declining VD levels. ${ }^{4}$ Furthermore, a meta-analysis lately has indicated that VD deficiency increases the risk of prediabetes. ${ }^{5}$ Various processes justifying the role of VD in glucose regulation include its anti-inflammatory, immune balancing functions, ${ }^{6}$ direct stimulation of insulin secretion from pancreatic beta cells and an indirect effect by regulating intracellular calcium lev- els in beta cells and ensuing insulin release. ${ }^{7}$ Additionally, the expression of VD receptors in insulin-sensitive tissues like muscle, liver and adipose tissue substantiates its role in insulin sensitivity and glucose metabolism. ${ }^{8}$

Dyslipidemia is a cardinal threat for atherosclerosis and CVD. ${ }^{9}$ A longitudinal community-based study has shown the prospective association of deficient VD with high-density lipoprotein-cholesterol (HDL-C) and a higher ratio of total cholesterol (TC) and HDL-C. ${ }^{10}$ Severity of coronary atherosclerosis assessed in patients who underwent catheterization was found to have an inverse correlation with VD. ${ }^{11}$ As per one study low VD is considered as an independent risk factor of mortality due to CVD. ${ }^{12}$ This is attributed to its direct and indirect effects on lipid metabolism. By stimulating lipoprotein lipase in adipose tissue it directly reduces $\mathrm{TG}^{13}$ while its indirect effect is discerned by calcium up-regulation which

\section{Corresponding Author:}

Dr. Vidya Bhakta, Medical Laboratory, Dr Sulaiman Al Habib Medical Group, Olaya Medical Complex, P.O Box 91877; Riyadh 11643, KSA. Mobile: +966580718765; E-mail: vidya.bhakta@rediffmail.com

ISSN: 2231-2196 (Print) ISSN: 0975-5241 (Online)

Received: $04.07 .2021 \quad$ Revised: 12.08 .2021

Accepted: 15.09 .2021

Published: 13.12 .2021 
induces the formation of calcium fatty acid complexes in the intestine thereby reducing its absorption and increasing faecal fat excretion. ${ }^{14}$ Small and dense low-density lipoprotein (sd LDL) have a stronger predisposition to atherosclerosis compared to other lipid parameters. ${ }^{15}$ AIP is an excellent marker of sd LDL and well predicts CVD as shown in several studies. ${ }^{16,17}$ Current study aims to assess serum VD status in prediabetic patients and its relation with AIP.

\section{MATERIALS AND METHODS}

Retrospective collection of data was accomplished on first time diagnosed prediabetic patients in the age group of 18 65 years during the period January 2019 to December 2020. Prediabetes was diagnosed based on an HbA1C level of 5.7 - $6.4 \%{ }^{18}$ The selected patients also had concurrent lab investigations for lipid profile and vitamin $\mathrm{D}$. The tests were requested as part of routine investigations based on the clinical history and not done exclusively for the study purpose. Age, gender-matched normoglycemic control subjects were selected with $\mathrm{HbA} 1 \mathrm{c}<5.7 \%$ in the same duration. Exclusion criteria were previous history of diabetes, malignancies, other chronic illnesses, diseases that affect VD levels like thyroid, parathyroid disorders, Cushing syndrome etc. Those with a history of VD supplementation and those with incomplete lab data were also eliminated. Age, gender of subjects along with other information was retrieved from health records and entered on a Microsoft excel sheet with proper coding while keeping the identity of all participants strictly confidential. Ethical approval was waived due to the retrospective nature of the study.

\section{Anthropometric and laboratory parameters}

Height, weight, systolic and diastolic blood pressures of all subjects were noted. Body mass index was calculated as Height / (weight $)^{2}\left(\mathrm{~kg} / \mathrm{m}^{2}\right)$.

The laboratory tests were performed after fasting of 8 hours and tests included were fasting blood sugar (FBS), HbA1C, $\mathrm{TC}$, triglycerides (TG), low-density lipoprotein cholesterol (LDL-C), HDL-C, 25(OH) VD. All lab parameters were measured on an Alinity ci analyzer, Abbott, Germany. For study purposes VD levels were categorized as Deficient, $25(\mathrm{OH}) \mathrm{D}<50 \mathrm{nmol} / \mathrm{L}$; Insufficient, $50-75 \mathrm{nmol} / \mathrm{L}$ and Sufficient, $\geqq 75 \mathrm{nmol} / \mathrm{L} .{ }^{19} \mathrm{AIP}$ was calculated as the logarithm to the base 10 of the ratio TG / HDL-C and value $>0.1$ was considered to be associated with increased cardiovascular risk. ${ }^{17}$

\section{Statistical Analysis}

Statistical analysis was performed using SPSS 20.0 version. Descriptive statistics were presented as frequency, the percentage for qualitative variables and mean with standard deviation for numerical variables respectively. For inferential statistics, categorical variables were compared by chi-square analysis and continuous variables by t-test and analysis of variance (ANOVA). The variables were compared in prediabetic and normoglycemic groups, stratified analysis for two groups under three categories of VD; deficient, insufficient and sufficient. Pearsons correlation coefficients were deduced for all variables with $\mathrm{HbA1c}$ and AIP among prediabetic subjects. Regression analysis was performed for the risk of prediabetes and higher AIP $(>0.1)$. According to VD categories and model adjustments were done for age, gender and BMI. Statistical significance was defined as p-value $<0.05$.

\section{RESULTS}

Table 1 summarizes the clinical and biochemical variables of all subjects. Means of TG, TC, LDL-C, were significantly higher while the mean of HDL-C was significantly lower in the prediabetes group. Mean AIP in prediabetic group was $0.13 \pm 0.30$ whereas in control group it was significantly lower, $-0.049 \pm .245$. VD deficiency was significantly more prevalent in the prediabetic group (56.9\%) compared to the control group (43.1\%). The mean VD level in prediabetic subjects was $57.9 \pm 20.8 \mathrm{nmol} / 1$ while in the control group it was $63.27 \pm 23.7 \mathrm{nmol} / \mathrm{L}$.

Tables 2, 3 show stratified analysis of variables in prediabetic and control groups based on VD level. A significant increasing trend was noticed in BMI with decreasing VD in the prediabetes group. Additionally, in the prediabetic group HbA1c, TG, TC, LDL-C exhibited distinct increasing tendency and HDL -C showed decreasing bent analogous to reducing VD level categories with statistical significance. AIP was highest in deficient VD category in prediabetes group $(0.35 \pm 0.19)$, lower in insufficient $(0.05 \pm 0.25)$ and lowest insufficient category $(-0.13 \pm 0.22)$ although no distinguishable trend was perceptible in control group.

Table 4 shows a positive correlation of AIP with BMI, HbA1c, TC, TG, LDL and a negative correlation with VD and HDL- $\mathrm{C}$ in the prediabetic population, with statistical significance. $\mathrm{HbA} 1 \mathrm{c}$ had a significant positive correlation with BMI, TC, TG and AIP and a negative correlation with VD and HDL-C among prediabetic subjects.

Table 5 depicts logistic regression showing that the risk of prediabetes dropped to 0.513 times in the insufficient category and 0.673 times in the sufficient category when compared to the VD deficient category (considered as reference). The risk lessened with rising VD levels even after adjusting for age, gender and BMI. As for increased cardiovascular risk in prediabetic patients, based on AIP $>0.1$, the

The risk was 0.101 times and 0.015 times lower in insufficient and sufficient categories as related to the risk in deficient categories. The risk still showed a similar downswing when adjusted for age, gender and BMI. 


\section{DISCUSSION}

There has been an augmented indication of the protective role of VD against several metabolic disorders including prediabetes and CVD. The current study affirms that VD inadequacy is associated with prediabetes and increased cardiovascular risk. Additionally, in prediabetic patients who are already predisposed to CVD, the threat is further exaggerated due to VD deficiency.

Based on a recent study, metabolic risk factors are accentuated in prediabetes due to VD inadequacy.$^{20}$ Keeping up with previous findings, the study showed inverse association of BMI and 25(OH)D with a clear increasing trend of BMI with decreasing $25(\mathrm{OH}) \mathrm{D}$ level in prediabetic patients. ${ }^{21}$ The linkage is explained by VD receptor polymorphism which when expressed on preadipocytes leads to increased adiposity. ${ }^{22}$ Other experiments suggest that by stimulating parathormone, VD deficiency causes more influx of calcium into adipocytes and hence lipogenesis. ${ }^{23}$ As an indicator of cardiovascular risk, AIP was significantly higher in prediabetes in the current study similar to a previous one that also emphasized on early identification of prediabetes to prevent CVD. ${ }^{24}$ Standing by our findings, previous studies have shown a similar association of VD and lipids ${ }^{25,26}$ Several mechanisms have been proposed; firstly by modulating the Apolipoprotein A-1 levels VD regulates cholesterol transportation, secondly by increasing lipoprotein lipase gene expression it enhances clearance of lipoprotein particles. Additionally, 25(OH)D induced hyperparathyroidism leads to decreased peripheral TG clearance and a rise in hepatocellular $\mathrm{Ca}^{2+}$ stimulates microsomal triglyceride transfer protein leading to hypertriglyceridemia. It may also reduce the formation of foam cells hence increasing HDL-C. 25(OH)D is also shown to suppress sterol regulatory element-binding proteins (SREBPs), associated with lipogenesis of cholesterol and triglycerides, by proteolysis and ubiquitin-mediated degradation of SREBP cleavage activating protein (SCAP) ${ }^{27,28}$ Besides, VD indirectly influences lipid metabolism by affecting insulin resistance and inflammation. ${ }^{?}$

AIP exhibited an increasing trend with falling VD categories with statistical significance in prediabetic subjects, but not in the control group. This was an additional finding to the previous claim that subjects with prediabetes have worse cardiometabolic risk profiles as compared to those with normoglycemia which is shown in the present study as well. Similarly, HbA1C showed a rising tendency from sufficient to deficient VD categories in the prediabetic group. VD showed a negative correlation with $\mathrm{HbA1c}$ and AIP which corroborates with previous studies 5,25 . Further, regression analysis divulged an increasing risk of prediabetes with diminishing VD level as also proven in a recent meta-analysis? .

There were certain limitations in the present study that are noteworthy while considering the results. Firstly, it was a retrospective data collection and selection bias could not be avoided. Calcium and parathormone levels were not measured which could have possibly impacted the association of Vitamin D and lipids. Furthermore, it was a small scale study which might have affected the statistical power. Nevertheless, the study found a significant association between $\mathrm{Vi}$ tamin D status and AIP in prediabetic patients but was not evident in normoglycemic subjects.

\section{CONCLUSION}

To summarize, Vitamin D levels are lower in prediabetic subjects and are inversely related to HbA1c and the Atherogenic index of plasma. Vitamin D inadequacy in prediabetes poses a cardiovascular risk. Since these patients have an equal propensity for progression to various complications of type 2 diabetes mellitus it would be worth knowing Vitamin D status and intervening timely with supplementation if needed which would enable to improve future prognosis and perhaps better quality of life.

\section{Source of Funding: Nil}

\section{ACKNOWLEDGEMENTS}

The authors acknowledge the immense help received from the scholars whose articles are cited and included in the manuscript. The authors are also grateful to authors/editors/publishers of all those articles, journals from where the literature for this article has been reviewed and discussed.

\section{Conflict of Interest: None}

\section{REFERENCES}

1. Mitri J, Muraru MD, Pittas AG. Vitamin D and type 2 diabetes: a systematic review.Eur J Clin Nutr2011 ; 65(9): 1005 -15.

2. Huang Y, Cai X, Mai W, Li M, Hu Y. Association between prediabetes and risk of cardiovascular disease and all-cause mortality: systematic review and meta-analysis. Bri Med J. 2016; 355.

3. Gupta AK, Brashear MM, Johnson WD. Prediabetes and prehypertension in healthy adults are associated with low vitamin D levels. Diabetes Care. 2011; 34(3): 658-60.

4. Deleskog A, Hilding A, Brismar K, Hamsten A, Efendic S, Östenson CG. Low serum 25-hydroxyvitamin D level predicts progression to type 2 diabetes in individuals with prediabetes but not with normal glucose tolerance. Diabetologia. 2012 (6):1668-78.

5. Yu L, Zhai Y, Shen S. Association between vitamin D and prediabetes: a PRISMA-compliant meta-analysis. Medicine. 2020; 99(8)

6. Vanherwegen AS, Gysemans C, Mathieu C. Regulation of immune function by vitamin D and its use in diseases of immunity. Clin Endocrinol Metab. 2017;46(4):1061-94.

7. Alvarez JA, Ashraf A. Role of vitamin D in insulin secretion and insulin sensitivity for glucose homeostasis.Int J Endocrinol 2010 Jan;2010. 
8. Sung CC, Liao MT, Lu KC, Wu CC. Role of vitamin D in insulin resistance. J Biomed Biotechnol2012 Oct;2012.

9. Andersson C, Lyass A, Vasan RS, Massaro JM, D’AgostinoSr RB, Robins SJ. Long-term risk of cardiovascular events across a spectrum of adverse major plasma lipid combinations in the Framingham Heart Study. Am Heart J. 2014; 168(6): 878-83.

10. Faridi KF, Zhao D, Martin SS, Lupton JR, Jones SR, Guallar E. Serum vitamin D and change in lipid levels over $5 \mathrm{y}$ : The Atherosclerosis Risk in Communities study. Nutrition. 2017; 38 : 85-93.

11. Dziedzic EA, Przychodzeń S, Dąbrowski M. The effects of vitamin D on severity of coronary artery atherosclerosis and lipid profile of cardiac patients. Arch Med Sci. 2016; 12(6):1199.

12. Dobnig H, Pilz S, Scharnagl H, Renner W, Seelhorst U, Wellnitz $\mathrm{B}$ et al. Independent association of low serum 25-hydroxyvitamin D and 1, 25-dihydroxy vitamin D levels with all-cause and cardiovascular mortality.Arch Intern Med 2008;168(12):1340-9.

13. Wang JH, Kerala T, Solakivi T, Minasyan A, Kalueff AV, Tuohimaa P. Serum cholesterol and expression of ApoAI, LXR $\beta$ and SREBP2 in vitamin D receptor knock-out mice. J Steroid BiochemMol Biol 2009; 113(3-5): 222-6.

14. Christensen R, Lorenzen JK, Smith CR, Bartels EM, Melanson EL, Saris WH et al. Effect of calcium from dairy and dietary supplements on faecal fat excretion: a meta-analysis of randomized controlled trials. Obes Rev. 2009;10(4):475-86.

15. Ji Y, Bai C. Research progress of hypertriglyceridemia and coronary heart disease. Heart and Mind. 2018 ; 2(2):40.

16. On A, Can G, Kaya H, Hergenç G. "Atherogenic index of plasma" $(\log 10$ triglyceride/high-density lipoprotein- cholesterol) predicts high blood pressure, diabetes, and vascular events. J Clin Lipidol.2010;4(2):89-98.

17. Dobiasova M. AIP--atherogenic index of plasma as a significant predictor of cardiovascular risk: from research to practice. VnitrLek. 2006;52(1):64-71.

18. Pippitt K, Li M, Gurgle HE. Diabetes mellitus: screening and diagnosis.AmFam Physician 2016; 93(2): 103 -9.
19. Holick MF. Vitamin D deficiency. N Engl J Med. 2007; 357(3): 266-81.

20. Tian LQ, Shi WQ, Zhou Y, Zhang YW, Zhang ML. The Association of serum vitamin $\mathrm{D}$ deficiency and metabolic risk factors in Chinese adults with prediabetes: a cross-sectional study. J Nutr Sci Vitaminol. 2019;65(3):211-8.

21. Yao Y, Zhu L, He L, Duan Y, Liang W, Nie Z et al. A metaanalysis of the relationship between vitamin D deficiency and obesity. Int J Clin Exp Med. 2015;8(9):14977.

22. Wong KE, Kong J, Zhang W, Szeto FL, Ye H, Deb DK et al. Targeted expression of human vitamin $\mathrm{D}$ receptor in adipocytes decreases energy expenditure and induces obesity in mice. J Biol Chem. 2011 286(39):33804-10.

23. Hjelmesæth J, Hofsø D, Aasheim ET, Jenssen T, Moan J, Hager $\mathrm{H}$ et al. Parathyroid hormone, but not vitamin $\mathrm{D}$, is associated with the metabolic syndrome in morbidly obese women and men: a cross-sectional study. Cardiovasc Diabetol. 2009; (1):17.

24. Hu H, Mizoue T, Sasaki N, Ogasawara T, Tomita K, Nagahama $\mathrm{S}$ et al. Prediabetes and cardiovascular disease risk: a nested case-control study. Atherosclerosis. 2018;278:1-6.

25. Wang Y, Si S, Liu J, Wang Z, Jia H, Feng K. The associations of serum lipids with vitamin D status. PLoS One. 2016 ;11(10):157-165

26. Mai XM, Videm V, Sheehan NA, Chen Y, Langhammer A, Sun YQ. Potential causal associations of serum 25-hydroxyvitamin D with lipids: a Mendelian randomization approach of the HUNT study. Eur J Epidemiol 2019;34(1):57-66.

27. Kumar S, Nanduri R, Bhagyaraj E, Kalra R, Ahuja N, Chacko AP, et al. Vitamin D3-VDR-PTPN6 axis mediated autophagy contributes to the inhibition of macrophage foam cell formation. Autophagy. 2020; 25:1-7.

28. Asano L, Watanabe M, Ryoden Y, Usuda K, Yamaguchi T, Khambu B, et al. Vitamin D metabolite, 25-hydroxyvitamin D, regulates lipid metabolism by inducing degradation of SREBP/ SCAP. Cell Chem Biol 2017;24(2): 207-17.

Table 1: Clinical and Biochemical parameters of all subjects

\begin{tabular}{|c|c|c|c|}
\hline & Normoglycemic $(n=299)$ & Prediabetic $(\mathrm{n}=\mathbf{2 7 0})$ & p value \\
\hline Age(years) & $35.71+8.43$ & $44.98+9.40$ & $<0.001^{*}$ \\
\hline Gender(Female/Male) & $142 / 157$ & $134 / 136$ & 0.610 \\
\hline BMI $\left(\mathrm{kg} / \mathrm{m}^{2}\right)$ & $22.79+1.92$ & $25 \cdot 53+2.89$ & $<0.001^{*}$ \\
\hline Systolic BP(mm Hg) & $130.79+8.21$ & $135.53+11.09$ & $<0.001^{*}$ \\
\hline Diastolic BP(mm Hg) & $78.02+6.59$ & $79.12+9.11$ & 0.097 \\
\hline $\mathrm{HbAlC}(\%)$ & $4.89+0.29$ & $5.93+0.19$ & $<0.001^{*}$ \\
\hline FBS (mmol/L) & $6.50+0.27$ & $5.41+0.59$ & 0.521 \\
\hline TotalCholesterol (mmol/L) & $4.75+0.81$ & $5.17+0.79$ & $<0.001^{*}$ \\
\hline $\mathrm{LDL}-\mathrm{C}(\mathrm{mmol} / \mathrm{L})$ & $2.6389+0.86828$ & $2.34+0.89$ & $<0.001^{*}$ \\
\hline $\mathrm{HDL}-\mathrm{C}((\mathrm{mmol} / \mathrm{L})$ & $1.4477+0.39888$ & $1.15+0.31$ & $<0.001^{*}$ \\
\hline Vitamin D (nmol/L) & $63.2742+23.78350$ & $57 \cdot 91+20.83$ & $0.005^{*}$ \\
\hline Deficient (\%) & $81(43.1 \%)$ & $107(56.9 \%)$ & \\
\hline Insufficient(\%) & $146(59.6 \%)$ & $99(40.4 \%)$ & $0.003^{*}$ \\
\hline Sufficient(\%) & $72(52.9 \%)$ & $64(47.1 \%)$ & \\
\hline AIP & $-0.049+.245$ & $0.130+.298$ & $<0.001^{*}$ \\
\hline
\end{tabular}

Continuos data presented as Mean + standard deviation and categorical data as frequency (\%)/ proportion. p-value calculated using t-test and chi-square analysis; $\left(^{*}\right)$ p-value significant BMI: Body Mass Index; BP: blood pressure, HbAic: glycosylated haemoglobin; FBS: fasting blood sugar, LDL-C: low density lipoprotein-cholesterol HDL-C:: high-density lipoprotein-cholesterol, AIP: atherogenic index of plasma 
Table 2: Clinical and Biochemical parameters of Prediabetic subjects under Vitamin D Categories.

\begin{tabular}{|c|c|c|c|c|}
\hline & $\begin{array}{l}\text { Deficient VD } \\
(<50 \mathrm{nmol} / \mathrm{L})\end{array}$ & $\begin{array}{l}\text { InsufficientVD } \\
(50-74.9 \mathrm{nmol} / \mathrm{L})\end{array}$ & $\begin{array}{l}\text { Sufficient VD } \\
(>=75 \mathrm{nmol} / \mathrm{L})\end{array}$ & p value \\
\hline Age & $43.71+9.45$ & $44 \cdot 73+8.86$ & $47 \cdot 51+9 \cdot 77$ & $0.038^{*}$ \\
\hline Gender(Female/Male) & $61 / 46$ & $50 / 49$ & $23 / 41$ & $0.028^{*}$ \\
\hline BMI $\left(\mathrm{kg} / \mathrm{m}^{2}\right)$ & $26.69+3.04$ & $25.07+2.78$ & $24.31+2.02$ & $<0.001^{*}$ \\
\hline Systolic BP (mm Hg) & $136.25+12.20$ & $135.90+11.04$ & $133.75+9.00$ & 0.700 \\
\hline Diastolic BP (mm Hg) & $80.16+8.38$ & $78.93+10.38$ & $77.68+8.03$ & 0.273 \\
\hline Median Vitamin D & $38.65+7.19$ & $59 \cdot 49+6.87$ & $87.65+12.69$ & $<0.001^{*}$ \\
\hline $\mathrm{HbAlC}(\%)$ & $6.0+0.21556$ & $5.90+0.17$ & $5.86+0.14$ & $<0.001^{*}$ \\
\hline FBS (mmol/L) & $5 \cdot 50+0.56$ & $5.36+0.57$ & $5.34+0.64$ & 0.097 \\
\hline Triglycerides (mmol/L) & $2.1+0.50$ & $1.51+0.61$ & $1.08+0.45$ & $<0.001^{*}$ \\
\hline TotalCholesterol(mmol/L) & $5.53+0.65$ & $5.10+0.76$ & $4.68+0.74$ & $<0.001^{*}$ \\
\hline $\mathrm{LDL}$ C(mmol/L) & $2.58+0.86$ & $2.32+0.96$ & $1.99+0.72$ & $<0.001^{*}$ \\
\hline HDL C(mmol/L) & $0.93+0.27$ & $1.25+0.28$ & $1.37+0.19$ & $<0.001^{*}$ \\
\hline AIP & $0.35+0.19$ & $0.05+0.25$ & $-0.13+0.22$ & $<0.001^{*}$ \\
\hline
\end{tabular}

Continuous data presented as Mean +-standard deviation and categorical data as frequency (\%) /proportion, p-value Calculated from one way ANOVA; $\left({ }^{*}\right)$ p-value significant.

VD Vitamin D; BMI: Body mass Index; BP: blood pressure, HbA1c: glycosylated haemoglobin; FBS: fasting blood

Sugar, LDL C: low-density lipoprotein cholesterol HDL: high-density lipoprotein cholesterol, AIP: atherogenic index of plasma

Table 3: Clinical and Biochemical variables of Normoglycemic subjects under Vitamin D Categories.

\begin{tabular}{|c|c|c|c|c|}
\hline & $\begin{array}{l}\text { Deficient VD } \\
(<50 \mathrm{nmol} / \mathrm{L})\end{array}$ & $\begin{array}{l}\text { Insufficient VD } \\
(50-74 \cdot 9 \mathrm{nmol} / \mathrm{L})\end{array}$ & $\begin{array}{l}\text { Sufficient VD } \\
(>=75 \mathrm{nmol} / \mathrm{L})\end{array}$ & p-value \\
\hline Age & $33.81+8.18$ & $35 \cdot 36+8.24$ & $38.62+8.44$ & $0.001^{*}$ \\
\hline Gender(F/M) & $42 / 39$ & $59 / 87$ & $41 / 31$ & $0.047^{*}$ \\
\hline $\operatorname{BMI}\left(\mathrm{kg} / \mathrm{m}^{2}\right)$ & $22.48+1.75$ & $22.87+1.97$ & $22.99+1.99$ & 0.310 \\
\hline Systolic BP(mmHg) & $129 \cdot 59+7 \cdot 53$ & $130.70+7.69$ & $132.34+9.70$ & 0.293 \\
\hline Diastolic BP(mmHg) & $77.86+6.37$ & $77.95+6.36$ & $78.34+7 \cdot 34$ & 0.879 \\
\hline MedianVitaminD(nmol/L) & $38.36+8.93$ & $61.33+7.30$ & $95.23+20.59$ & $<0.001^{*}$ \\
\hline $\mathrm{HbAlC}(\%)$ & $4.91+0.29$ & $4.88+0.30$ & $4.88+0.25$ & 0.470 \\
\hline $\mathrm{FBS}(\mathrm{mmol} / \mathrm{L})$ & $10.85+53.56$ & $4.85+0.40$ & $4.95+0.40$ & 0.139 \\
\hline Triglycerides(mmol/L) & $1.49+0.58$ & $1.30+0.57$ & $1.32+0.52$ & 0.053 \\
\hline TotalCholesterol(mmol/L) & $4.99+0.69$ & $4.65+0.83$ & $4.69+0.85$ & $0.009^{*}$ \\
\hline LDL-C(mmol/L) & $2.69+0.88$ & $2.59+0.86$ & $2.66+0.86$ & 0.672 \\
\hline HDL-C(mmol/L) & $1.50+0.48$ & $1.46+0.34$ & $1.35+0.37$ & $0.020^{*}$ \\
\hline AIP & $-0.015+0.26$ & $-0.080+0.24$ & $-0.026+0.21$ & 0.161 \\
\hline
\end{tabular}

Continuous data presented as Mean + standard deviation and categorical data as frequency (\%) / proportion, p-value calculated from one way ANOVA; $\left(^{*}\right)$ p-value significant.

VD: Vitamin D; BMI: Body mass Index; BP: blood pressure, HbA1c: glycosylated haemoglobin; FBS: fasting Blood sugar, LDL-C: low-density lipoprotein-Cholesterol; HDL-C: high-density lipoprotein cholesterol AIP: Atherogenic index of plasma 
Table 4: Correlation between HbAic and AIP with other variables

\begin{tabular}{|c|c|c|c|}
\hline \multirow{3}{*}{ Age } & & Hbaic (\%) & AIP \\
\hline & $\mathbf{r}$ & 0.08 & -0.109 \\
\hline & p value & 0.187 & 0.074 \\
\hline \multirow[t]{2}{*}{$\mathrm{BMI}(\mathrm{kg} / \mathrm{m} 2)$} & $r$ & 0.362 & 0.480 \\
\hline & $\mathrm{p}$ value & $<0.001$ & $<0.001^{*}$ \\
\hline \multirow[t]{2}{*}{$\mathrm{FBS}(\mathrm{mmol} / \mathrm{L})$} & $\mathrm{r}$ & 0.298 & 0.115 \\
\hline & p value & $<0.001$ & 0.058 \\
\hline \multirow[t]{2}{*}{ Vitamin D (nmol/L) } & $\mathrm{r}$ & -0.230 & -0.639 \\
\hline & $\mathrm{p}$ value & $<0.001$ & $<0.001^{*}$ \\
\hline \multirow[t]{2}{*}{ TotalCholestro $1(\mathrm{mmol} / \mathrm{L})$} & $\mathrm{r}$ & 0.196 & 0.584 \\
\hline & $\mathrm{p}$-value & 0.001 & $<0.001^{*}$ \\
\hline \multirow[t]{2}{*}{ Triglycerides (mmol/L) } & $\mathrm{r}$ & 0.266 & 0.897 \\
\hline & p value & $<0.001$ & $<0.001^{*}$ \\
\hline \multirow[t]{2}{*}{ HDL-C (mmol/L) } & $r$ & -0.348 & -0.828 \\
\hline & $\mathrm{p}$ value & $<0.001$ & $<0.001^{*}$ \\
\hline \multirow[t]{2}{*}{ LDL-C $(\mathrm{mmol} / \mathrm{L})$} & $\mathrm{r}$ & 0.071 & 0.349 \\
\hline & $\mathrm{p}$ value & $<0.001$ & $<0.001^{*}$ \\
\hline \multirow[t]{2}{*}{ AIP } & $r$ & 0.329 & \\
\hline & p value & $<0.001$ & \\
\hline
\end{tabular}

$\mathrm{r}$ : correlation coefficient; $\left({ }^{*}\right)$ p-value significant

BMI: Body mass Index; BP: blood pressure, HbAic: glycosylated

Haemoglobin; FBS: fasting blood sugar, LDL-C: low density

Lipoprotein-Cholesterol; HDL-C: high-density lipoprotein cholesterol

AIP: atherogenic index of plasma

Table 5: Regression Model to predict odds ratio for prediabetes and unfavourable AIP (>0.1).

\begin{tabular}{|c|c|c|c|c|c|}
\hline & $\begin{array}{l}\text { VD deficiency } \\
\text { OR }(95 \% \text { CI })\end{array}$ & $\begin{array}{c}\text { VD Insufficiency } \\
\text { OR }(95 \% \text { CI })\end{array}$ & p-value & $\begin{array}{l}\text { VD Sufficiency } \\
\text { OR }(95 \% \text { CI })\end{array}$ & p value \\
\hline \multicolumn{6}{|l|}{ Prediabetes } \\
\hline Unadjusted & 1.00 & $0.513(0.349-0.755)$ & $0.001^{*}$ & $0.673(0.432-1.048)$ & 0.080 \\
\hline Adjusted & 1.00 & $0.594(0.359-0.982)$ & 0.042 & $0.648(0.364-1.155)$ & 0.141 \\
\hline \multicolumn{6}{|l|}{$\operatorname{AIP}(>0.1)$} \\
\hline Unadjusted & 1.00 & $0.101(0.047-0.216)$ & $<0.001^{*}$ & 0.015 (0.005-0.039) & $<0.001^{*}$ \\
\hline Adjusted & 1.00 & $0.131(0.058-0.296)$ & $<0.001^{*}$ & 0.018 (0.006-0.053) & $<0.001^{*}$ \\
\hline
\end{tabular}

Model adjusted for age, gender, BMI (*) p-value significant.

OR: odds ratio; CI: confidence interval; AIP: atherogenic index of plasma 